\title{
Metabolic syndrome and insulin resistance in relation to biliary tract cancer and stone risks: a population-based study in
} Shanghai, China

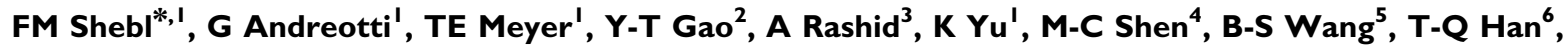 \\ B-H Zhang ${ }^{7}$, FZ Stanczyk ${ }^{8}$ and AW Hsing'
}

'Division of Cancer Epidemiology and Genetics, National Cancer Institute, National Institutes of Health, Rockville, MD, USA; ${ }^{2}$ Department of Epidemiology, Shanghai Cancer Institute, Shanghai, China; ${ }^{3}$ Department of Pathology, MD Anderson Cancer Center, Houston, TX, USA; ${ }^{4}$ Shanghai Tumor Hospital, Fudan University, Shanghai, China; ${ }^{5}$ Zhongshan Hospital, Fudan University, Shanghai, China; ${ }^{6}$ Ruijn Hospital, Shanghai Jiaotong University School of Medicine, Shanghai, China; ${ }^{7}$ Institute of Oriental Hepatobiliary Surgery, Second Military Medical University, Shanghai, China; ${ }^{8}$ Departments of Obstetrics and Gynecology and Preventive Medicine, Keck School of Medicine, University of Southern California, Los Angeles, CA, USA

BACKGROUND: Serum lipids, diabetes, and obesity, individual components of metabolic syndrome, are associated with biliary tract cancer and stone risk, but the associations of metabolic syndrome or insulin resistance with biliary tract cancers and stones are not well studied.

METHODS: In this population-based case-control study in Shanghai, China (627 biliary tract cancers, 1037 biliary stones, and 959 controls), metabolic syndrome was defined as the presence of any three of the five components, including high waist circumference, high triglycerides, low high-density lipoprotein cholesterol (HDL), high blood pressure, and diabetes. Insulin resistance and $\beta$-cell function were assessed, using homeostasis assessment models.

RESULTS: Metabolic syndrome was significantly associated with gallbladder cancer (odds ratio $(O R)=2.75$, 95\% confidence interval $(95 \% \mathrm{Cl})=1.82-4.15)$ and biliary stones $(\mathrm{OR}=1.64,95 \% \mathrm{Cl}=1.24-2.16)$, with a significant dose effect with increasing number of metabolic syndrome components $(P$ trend $<0.000 \mathrm{I})$. The observed association persisted among subjects without a history of diabetes. The association between insulin resistance and gallbladder cancer was borderline $(P$ trend $=0.06)$. There was a significant inverse association between $\beta$-cell function and gallbladder cancer risk $(P$ trend $<0.00 \mathrm{I})$.

CONCLUSION: Our findings suggest that metabolic syndrome and insulin resistance have a role in the aetiology of biliary tract cancers and biliary stones, and if confirmed, they imply that lifestyle control of these factors may lower the risk of biliary stones and biliary tract cancer.

British Journal of Cancer (201 I) 1 05, 1424- 1429. doi:I0.1038/bjc.201 I.363 www.bjcancer.com

Published online 13 September 201 I

(C) 201 I Cancer Research UK

Keywords: metabolic syndrome; insulin resistance; biliary tract cancers; biliary stones

Biliary tract cancers, including cancers of the gallbladder, extrahepatic bile duct, and ampulla of Vater, are rare but fatal malignancies (Hsing et al, 2006). Gallstones are undoubtedly the most important risk factor for biliary tract cancer (Hsing et al, 2006). Gallstones and biliary tract cancers have several risk factors in common, including obesity, diabetes, and hyperlipidaemia (Hsing et al, 2007, 2008; Ishiguro et al, 2008; Shebl et al, 2010), but the biologic mechanisms underlying the associations of obesity, diabetes, and hyperlipidaemia with gallstones and biliary tract cancers are not clear. These factors are components of metabolic syndrome, which is a cluster of related conditions (i.e., abdominal obesity, high blood pressure, high plasma glucose, high triglycerides, and decreased high-density lipoprotein cholesterol (HDL);

*Correspondence: Dr FM Shebl; E-mail: sheblf@mail.nih.gov Revised 6 July 2011; accepted 13 August 2011; published online 13 September 2011
(Cowey and Hardy, 2006)). While individual components of metabolic syndrome have been linked to biliary tract cancers and biliary stones (Maclure et al, 1989; Kodama et al, 1999; Tsai et al, 2004; Andreotti et al, 2008; Seidell, 2010), the total effect of metabolic syndrome on these two conditions has not been investigated fully.

To clarify further the role of metabolic syndrome in biliary tract cancer and biliary stones, we adapted a risk prediction approach to examine the associations of individual components and the collection of several components of metabolic syndrome with the risk of biliary tract cancers and biliary stones in a populationbased case-control study conducted in Shanghai, China. Further, since insulin resistance is an early process of metabolic syndrome, we also examined markers of insulin resistance (fasting serum levels of glucose and insulin, derived $\beta$-cell function (HOMA2$\% \beta$ ), and homeostasis insulin resistance (HOMA2-\%R)) with biliary tract cancer and stone risk in the same population. 


\section{MATERIALS AND METHODS}

\section{Study population}

Details of the study design and population have been reported elsewhere (Hsing et al, 2007, 2008). Briefly, between 1997 and 2001, patients newly diagnosed with primary biliary tract cancers (ICD9 code 156) were recruited using a rapid-reporting system in 42 hospitals in Shanghai. More than $95 \%$ of incident biliary tract cancer patients in Shanghai were enrolled into the study and $\sim 70 \%$ of cancer cases were confirmed by histopathologic assessment or medical records, and $30 \%$ were confirmed by clinical and imaging data. Biliary stone status among cancer cases was determined based on medical record review, self-report, or clinical evaluation (ultrasound and endoscopic retrograde cholangiopancreatography). Biliary stone patients without a history of cancer were identified from the same hospitals as cancer cases, confirmed using abdominal ultrasound, medical or surgical records, or pathologic specimens, and frequency-matched to the index cancer case on diagnosing hospital, age (5-year groups), and gender. Healthy subjects without biliary tract cancer were randomly selected from the Shanghai Resident Registry, and were frequency-matched to the index cancer case on gender and age (5-year groups). Biliary stone status among population controls was based on abdominal ultrasound ( $85 \%$ gave consent) or selfreport. Response rates for interviews were $95 \%$ among cases and $82 \%$ among eligible controls.

\section{Interview}

In-person interviews were conducted by trained interviewers using a structured questionnaire to obtain information on demographic characteristics, lifestyle factors, and medical conditions. Cancer and stone cases were interviewed within 3 weeks of diagnosis. Five percent of the subjects were re-interviewed 3 months after the initial interview to assess the reliability of the self-reported data. Concordance between the two interviews was $>90 \%$.

At interview, weight, height, and waist and hip circumference were measured. Physical measurements were taken twice. When the difference between the two measurements exceeded $a$ priori tolerance limits (height, $2 \mathrm{~cm}$; weight, $1 \mathrm{~kg}$; waist and hip circumference, $2 \mathrm{~cm}$ ), an additional measurement was taken and the average of the closest two values was used. We also used selfreported height and weight to calculate usual adult body mass index (BMI; $\mathrm{kg} \mathrm{m}^{-2}$ ) 5 years before interview. Diabetes and hypertension were based on self-reported information.

\section{Biologic sample collection and assessment}

Overnight fasting blood samples were collected from over $80 \%$ of the participants who gave consent. Within $4 \mathrm{~h}$ of collection, study personnel transported the samples to the central processing laboratory at the Shanghai Cancer Institute for processing, and samples were later shipped to the NCI repository on dry ice via Federal Express. Serum lipids were measured at the Laboratory of Biochemistry, Institute of Cardiovascular Diseases, Zhongshan Hospital, Shanghai Medical University (Fudan University). Assay kits for total cholesterol (oxidation enzymatic assay) (Smith et al, 1979), triglycerides (glycerol phosphoric acid oxidase assay) (Smith et al, 1979), and HDL (phosphotungstatemagnesium assay) (National Institutes of Health, 1995) were prepared by Shanghai No. 18 Pharmaceutical Company and used the spectrophotometer type 722 (Shanghai Third Analysis Instrument Company, Shanghai, China). LDL was calculated using the Friedewald formula (Rifai et al, 1992). Fasting blood glucose and human specific insulin were measured using commercially available radioimmunoassay kits (Linco Research,
St Charles, MO, USA) at the University of Southern California (FZS).

\section{Metabolic syndrome and insulin resistance components}

Metabolic syndrome was defined based on the criteria established by the Chinese Joint Committee for Developing Chinese Guidelines on Prevention and Treatment of Dyslipidemia in Adults (2007), which is similar to the criteria used by the American Heart Association/National Heart, Lung, and Blood Institute (Grundy et al, 2005), although the cutoffs are based on the distribution in the Chinese population and are lower than those used for Americans. An individual is classified as having metabolic syndrome if he/she has any three of these five conditions: high triglycerides $\left(\geqslant 1.7 \mathrm{mmoll}^{-1}\right)$, low HDL $\left(<1.04 \mathrm{mmol}^{-1}\right.$ for both men and women), high blood pressure (self-reported), diabetes (self-reported), and high waist circumference ( $\geqslant 90 \mathrm{~cm}$ in men and $\geqslant 80 \mathrm{~cm}$ in women).

For analyses of insulin resistance, we evaluated fasting serum levels of glucose and insulin, and using these measurements we derived a homeostasis assessment model 2 (HOMA2) for $\beta$-cell function (HOMA2-\% $\beta$ ) and insulin resistance (HOMA2-\%R) (models were estimated using software that is available at http:// www.dtu.ox.ac.uk/index.php?maindoc =/homa/; Levy et al, 1998). The HOMA2 is a method used to quantify insulin resistance and $\beta$-cell function. The higher the levels of HOMA2-\%R the higher the insulin resistance. The higher the levels of HOMA2- $\% \beta$ the higher the insulin sensitivity.

\section{Statistical analyses}

We examined each of the five components of metabolic syndrome individually, the total effect of metabolic syndrome (any three of the five components), and the degree of metabolic syndrome by calculating a $P$ trend for the number of components ranging from 0 to 5 factors.

Measurements of the insulin sensitivity markers were categorised into tertiles based on the distribution in the non-diabetic control population. Correlations between log-transformed insulin sensitivity markers were calculated using Spearman correlation coefficients. T-tests and Kruskal-Wallis statistics (when the assumption of normality was violated) were used for the bivariate comparison of cases and controls.

For all analyses, bile duct and ampulla of Vater cancers were compared with all population controls, gallbladder cancers were compared with population controls who did not have a cholecystectomy, and biliary stones cases were compared with population controls who did not have stones. All analyses were conducted using SAS 9.1 (SAS Institute, Cary, NC, USA). A total of 368 gallbladder, 191 extrahepatic bile duct, and 68 ampulla of Vater cancer cases, as well as 1037 patients with biliary stones, and 959 population controls were included in the metabolic syndrome analysis. The analysis of insulin resistance was restricted to subjects who donated blood samples and did not have a history of diabetes (self-reported) since diabetes and anti-diabetic medications can influence insulin sensitivity indices; this resulted in 219 cases with gallbladder cancer, 116 with extrahepatic bile duct cancer, with 926 biliary stones, and 553 population controls. Cases were frequency-matched; therefore, we used unconditional multivariable logistic regression to calculate odds ratios (ORs) and $95 \%$ confidence intervals (95\% CIs) adjusted for age, gender, and BMI. Body mass index was grouped according to the WHO classification for Asian populations where 18.5 to $<23 \mathrm{~kg} \mathrm{~m}^{-2}$ is considered normal, 23 to $<25 \mathrm{~kg} \mathrm{~m}^{-2}$ overweight, and $\geqslant 25 \mathrm{~kg} \mathrm{~m}^{-2}$ obese (WHO/IASO/IOTF, 2000; WHO Expert Consultation, 2004). To reduce the impact of cancer-related wasting, we excluded individuals who reported $>10 \%$ weight loss during the 5 years before cancer diagnosis. 


\section{RESULTS}

Associations of individual metabolic syndrome components with biliary tract cancer and biliary stone risk are shown in Table 1. Results for the ampulla of Vater cancers are not included due to small numbers. After adjustment for age, gender, and BMI, high serum triglycerides $\left(\geqslant 1.7 \mathrm{mmol}^{-1}\right)$ were associated with excess risks of gallbladder cancer $(\mathrm{OR}=2.0,95 \% \mathrm{CI}=1.48-2.70)$, extrahepatic bile duct cancer $(\mathrm{OR}=5.28,95 \% \mathrm{CI}=3.56-7.82)$, and biliary stones $(\mathrm{OR}=1.56,95 \% \mathrm{CI}=1.24-1.98)$, while low

Table I Odds ratios (ORs) and 95\% confidence intervals (Cls) for biliary tract cancer and biliary stones in relation to metabolic syndrome and its individual components

Biliary tract cancers

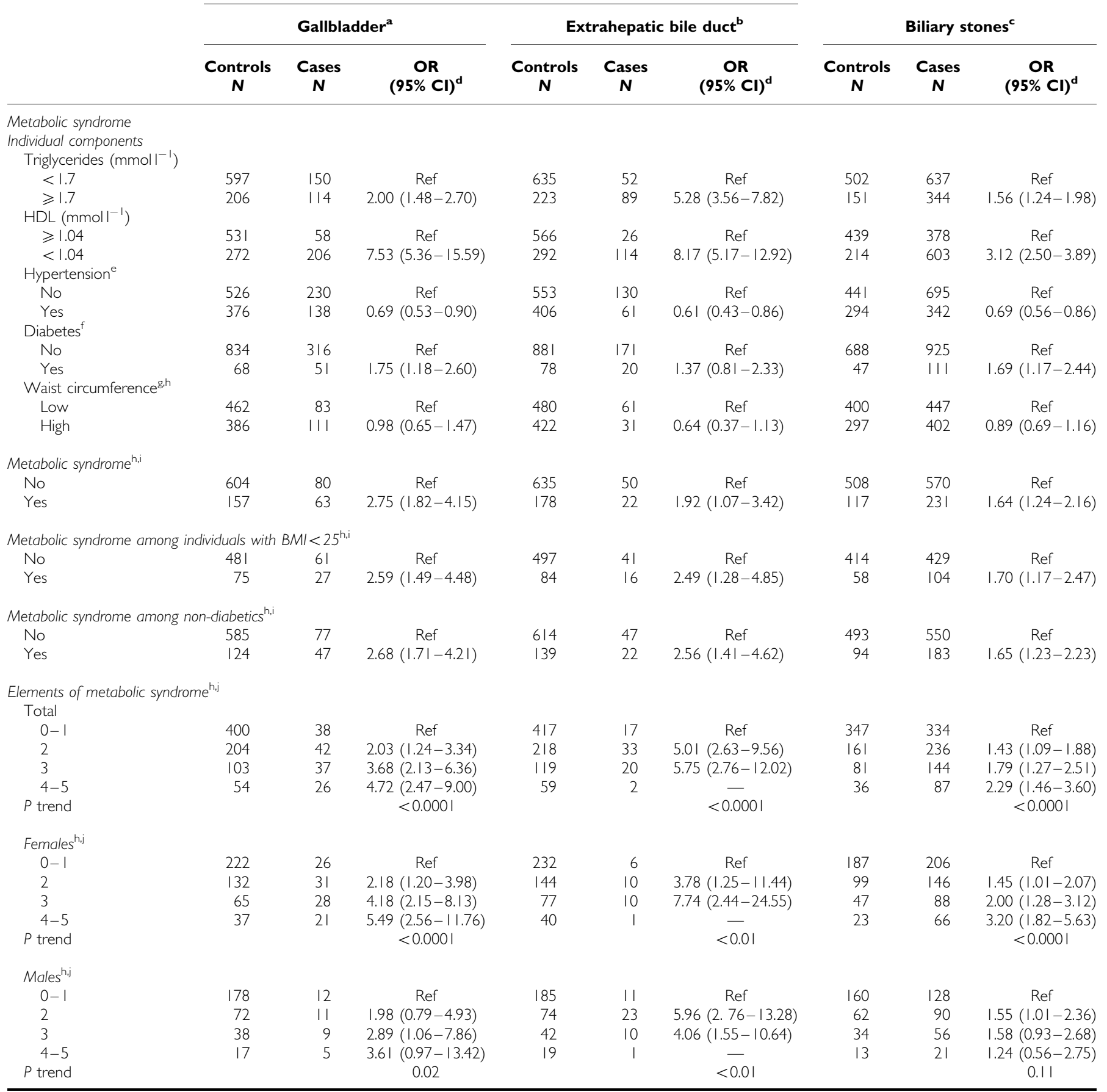

Abbreviations: $\mathrm{BMI}=$ body mass index; $\mathrm{HDL}=$ high-density lipoprotein cholesterol. ${ }^{\mathrm{a}}$ Gallbladder cancer cases were compared with population controls without history of prior cholecystectomy. 'Extrahepatic bile duct and ampulla of Vater cancer cases were compared with all population controls; ampulla of Vater cancer analyses are not shown due to small cell sizes. 'Individuals with gallstones were compared with controls without gallstones. 'Separate models were run for metabolic syndrome, metabolic syndrome score and

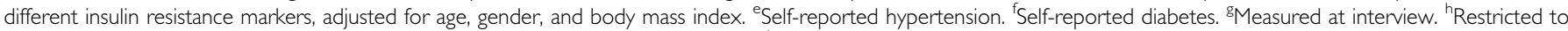
individuals who did not lose $>10 \%$ of weight within the 5 years before the interview. 'Based on Chinese Joint Committee for Developing Chinese Guidelines on Prevention and

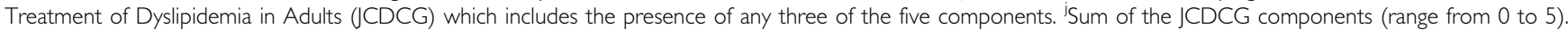


serum HDL was associated with excess risks of gallbladder cancer $(\mathrm{OR}=7.53,95 \% \mathrm{CI}=5.36-15.59)$, extrahepatic bile duct cancer $(\mathrm{OR}=8.17,95 \% \mathrm{CI}=5.17-12.92)$, and biliary stones $(\mathrm{OR}=3.12$, $95 \% \mathrm{CI}=2.50-3.89$ ). Diabetes (self-reported) was also associated with excess risks of gallbladder cancer and biliary stones $(\mathrm{OR}=1.95,95 \% \mathrm{CI}=1.18-2.60 ; \mathrm{OR}=1.69,95 \% \mathrm{CI}=1.17-2.44$, respectively). Hypertension had a significant inverse association with all three biliary diseases, while the effect of waist circumference was null.

Metabolic syndrome (any three of the five components) was significantly associated with increased risks of all three cancers: gallbladder cancer $(\mathrm{OR}=2.75,95 \% \mathrm{CI}=1.82-4.15)$, bile duct cancer $(\mathrm{OR}=1.92,95 \% \mathrm{CI}=1.07-3.42)$, and biliary stones $(\mathrm{OR}=1.64,95 \% \mathrm{CI}=1.24-2.16)$, after adjustment for age, gender, and BMI (Table 1). Similar risk estimates persisted when the analysis was restricted to individuals with $\mathrm{BMI}<25$ or nondiabetics (metabolic syndrome defined as any three of the remaining four components). The risks of gallbladder cancer, bile duct cancer, and biliary stones increased with increasing number of metabolic syndrome components ( $P$ for trend $<0.0001$ ), with those having 4-5 components having risks as high as 4.5 -fold when compared with those having 0-1 components (Table 1). Similar risk estimates were seen, regardless of BMI status, or gallstone status. We observed more pronounced associations in women than in men.

Associations for markers of insulin resistance among individuals without a history of diabetes are shown in Table 2. After adjustment for age, gender, and BMI, high fasting glucose was significantly associated with significant excess risk of gallbladder and bile duct cancer and biliary stones. Participants in the highest tertile of HOMA2- $\% \beta$ (least insulin resistant) had a reduced risk of gallbladder cancer $(\mathrm{OR}=0.38,95 \% \mathrm{CI}=0.24-0.58)$, bile duct cancer $(\mathrm{OR}=0.38,95 \% \mathrm{CI}=0.21-0.67)$, and biliary stones
$(\mathrm{OR}=0.70,95 \% \mathrm{CI}=0.51-0.97)$ compared with individuals in the lowest tertile of HOMA2- $\% \beta$ (most insulin resistant). Fasting insulin and HOMA2-\%R were not significantly associated with biliary tract cancers or stones.

Correlations among the metabolic and insulin sensitivity biomarkers were high among non-diabetic controls (Supplementary Table 1). HOMA2- $\% \beta$ was positively correlated with BMI and stones. HOMA2-\%R was positively correlated with BMI, waist circumference, and stones. Serum levels of insulin and glucose were positively correlated with BMI, waist circumference, and stones.

\section{DISCUSSION}

In this population-based study, metabolic syndrome and insulin resistance (among those without a history of diabetes) were associated with excess risks of biliary tract cancer and stones. There was a dose-response relationship between increasing number of metabolic syndrome components and risk of biliary tract cancer and stones. The observed association between metabolic syndrome and biliary tract cancer and stones was more pronounced in women than in men, and it persisted among non-obese $(\mathrm{BMI}<25)$ and non-diabetic individuals.

Interestingly, the association between some components of metabolic syndrome, such as HDL, and cancer was stronger than that between metabolic syndrome (defined as having three or more of the five components) and cancer. This results because the definition of metabolic syndrome is stricter than the definition of the individual components, so that individuals have to have three or more components to be classified as having metabolic syndrome. Cancer patients who have only one or two of the components would not be classified as having metabolic syndrome. Therefore, the prevalence of metabolic syndrome is lower than that of each individual component in cancer patients,

Table 2 Odds ratios (ORs) and 95\% confidence intervals (Cls) for biliary tract cancer and gallstones in relation to insulin resistance/sensitivity markers among subjects without a history of diabetes

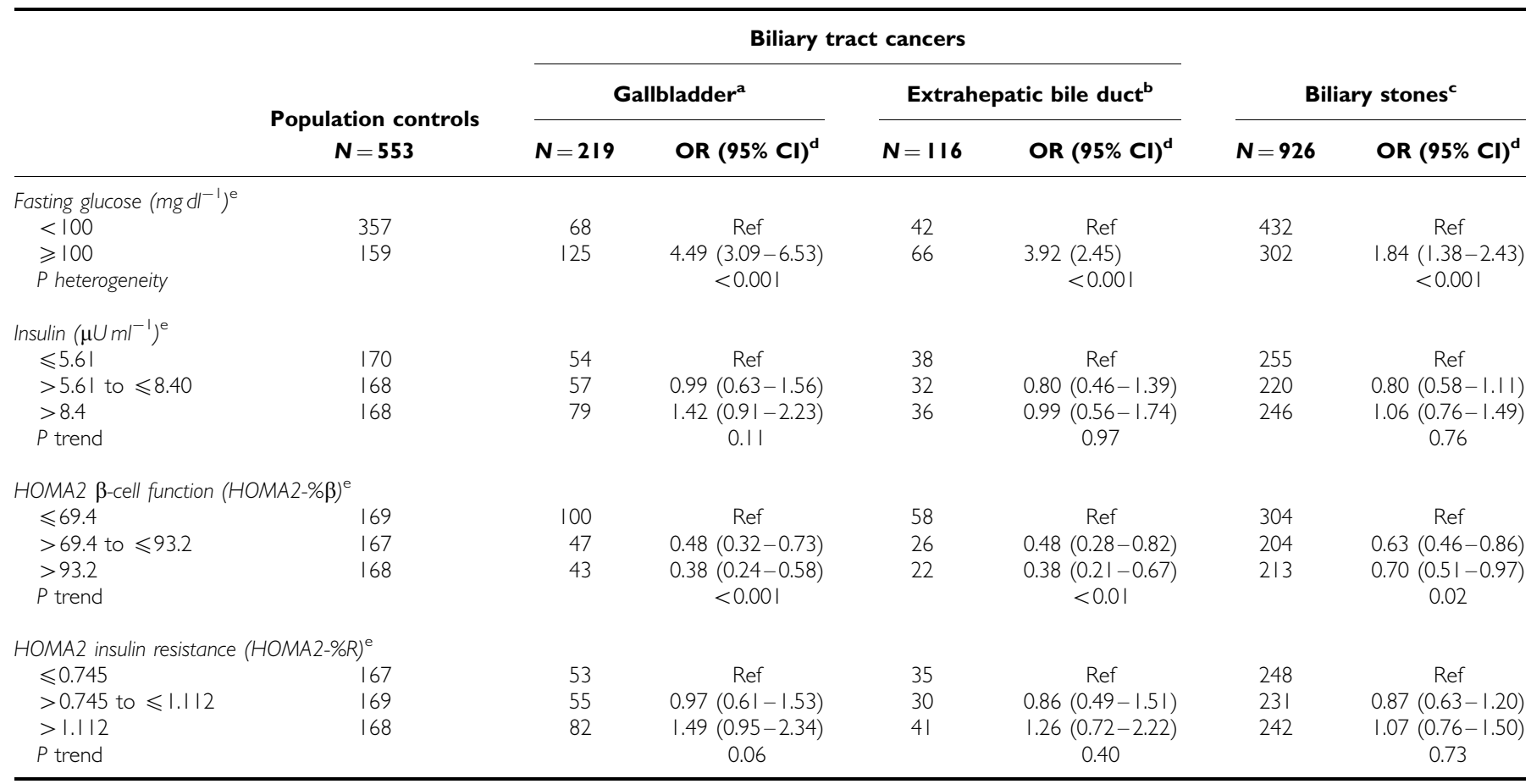

Abbreviation: HOMA2 = homeostasis assessment model 2. ${ }^{\mathrm{a}} \mathrm{G}$ allbladder cancer cases were compared with population controls without history of prior cholecystectomy. ${ }^{b}$ Extrahepatic bile duct cancer cases were compared with all population controls. Individuals with biliary stones were compared with controls without biliary stones. ${ }^{\mathrm{c}}$ Separate

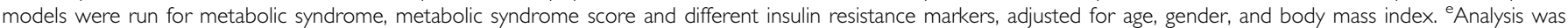
restricted to non-diabetics who had enough collected blood sample. 
resulting in smaller ORs for metabolic syndrome than for the individual components.

The observed association with metabolic syndrome does not appear to be explained entirely by a single component of the syndrome, since there was a significant dose-response relationship between the number of metabolic syndrome components and the risk of biliary tract cancer and stones. This dose-response relationship suggests that the presence of multiple components may have an additive effect on the risk of biliary tract cancers and stones. It is noteworthy that, although only three of the five individual components were positively associated with stone and cancer risks in the study, individuals having four or five components had the highest risk (4.5-fold). This observation suggests that the severity of metabolic syndrome exacerbates the development of stones and cancer.

The association of metabolic syndrome with biliary tract cancer and stones is expected and biologically plausible, as several of the components have been individually linked to stone and cancer risk in the study. For example, earlier we reported that, independent of BMI, diabetes was associated with a 2-fold risk of biliary stone and cancer, with biliary stones and low HDL accounting for $60 \%$ and $17 \%$ of the diabetes effect on gallbladder cancer risk (Shebl et al, 2010). It has also been shown that low serum HDL and high triglycerides result in oversaturation of cholesterol in bile and impaired gallbladder emptying, which leads to the formation of gallstones (Janowitz et al, 1992; Jonkers et al, 2003; Shor et al, 2008; Maurer et al, 2009; Tabet and Rye, 2009), and thereby to a higher risk of biliary tract cancer. Independent of stone formation, low serum HDL and high triglycerides are associated with increased LDL oxidation and formation of reactive oxygen species (ROS; Shor et al, 2008; Tabet and Rye, 2009), which in turn can increase oxidative DNA damage and promote cancer development (Jaiswal et al, 2001). Although hypertension was not individually positively associated with gallbladder cancer risk, the clustering of hypertension with other four components of metabolic syndrome was associated with a 4 -fold risk. The inverse association with hypertension needs to be replicated.

The lack of an association with waist circumference was somewhat surprising, but could be explained in part by the inherent limitation of the cross-sectional design of the study. Waist circumference in our study was measured at interview, which may have been affected by the presence of cancer (and weight loss), and may not represent usual adult waist size. Similarly, in our earlier analysis, BMI at interview was not associated with biliary tract cancer or stones, while usual adult BMI (5 years before interview) was associated with an excess risk (Hsing et al, 2008), suggesting that body size at interview (closer to the time of cancer diagnosis) may not reflect usual adult body size.

The observation that, among individuals without a history of diabetes, hyperglycaemia and impaired $\beta$-cell function were associated with biliary tract cancer and stone risk underscores the importance of insulin resistance, even in the pre-diabetes state. Hyperglycaemia, characterised by high fasting glucose and insulin levels, and impaired $\beta$-cell function are markers of insulin resistance. It has been shown that before the development of overt type 2 diabetes, insulin resistance, including conditions of hyperglycaemia, progressive deterioration of $\beta$-cell function, and hyperinsulinaemia, can exist for many years in a pre-diabetic stage (Lyssenko et al, 2005). Hyperglycaemia, in addition to being part of insulin resistance, is associated with increased oxidative stress, accumulation of advanced glycation end products, which in turn can lead to activation of NF- $\kappa \mathrm{B}$, formation of ROS, and tumourigenesis (Abe and Yamagishi, 2008).

Part of the biologic mechanism leading from insulin resistance and metabolic syndrome to the development of biliary tract cancers may be mediated by biliary stones. Adjustment for stones substantially attenuated the association between metabolic syn- drome and biliary tract cancer (data not shown), suggesting that stones may mediate part of the association between metabolic syndrome and cancer. It has been shown that inflammation has a key role in stone formation as well as in the pathogenesis of metabolic syndrome and insulin resistance (de Luca and Olefsky, 2008; Maurer et al, 2009; Olefsky and Glass, 2010). Therefore, it is likely that chronic inflammation may act as one of the mechanisms that underlie the association of obesity, diabetes, insulin resistance, and the metabolic syndrome with stones and biliary tract cancer. It should be noted that obesity, a common risk factor for diabetes, insulin resistance, and metabolic syndrome, is considered an inflammatory disease characterised by a state of chronic subclinical inflammation, which is involved in the pathogenesis of insulin resistance in the absence of overt diabetes (Bastard et al, 2006).

Strengths of our study include the relatively large size and minimal selection bias due to the population-based design and high response rate. Because subject selection and response are unrelated to the exposure of interest (i.e., metabolic syndrome) selection bias in our study should be minimal. In addition, misclassification of outcome (cancer and stones) is also minimal due to the detailed evaluation of cancer status from clinical and pathology reports combined with near complete assessment of stone status through careful review of medical records and ultrasound examinations. We also minimised the impact of confounding by adjusting for known confounders, such as age, gender, and BMI, although residual confounding may still exist. An example of possible residual confounding is treatment for dyslipidaemia. However, if individuals were on treatment they would have had lower levels of triglyceride, which would bias the association towards the null. Therefore, our observed association is conservative and is an underestimate of the effect of dyslipidaemia and metabolic syndrome.

Limitations of the study should be noted. Misclassification of biomarkers among cases, such as HDL, insulin, and glucose, is possible, since blood samples were taken at enrollment; therefore, the measurements in some cases may have been affected by weight loss among cases as a result of cancer. Although we are not able to remove the effect of reverse causation entirely, we attempted to minimise its impact by limiting the analysis to those with $<10 \%$ weight loss in the previous 5 years. Of note, due to the study design, we were unable to address the relationship between duration of metabolic syndrome and cancer/stone risk.

In summary, our study showed an increased risk of biliary tract cancer and biliary stones among individuals with metabolic syndrome and insulin resistance without a history of diabetes. These findings, if confirmed, suggest that lifestyle changes to control metabolic syndrome may help lower the risk of biliary stones and cancer.

\section{ACKNOWLEDGEMENTS}

We thank our collaborators namely, the surgeons and pathologists in Shanghai; Shelley Niwa of Westat for data management; and Chia-Rong Cheng, Lu Sun, and Kai Wu of the Shanghai Cancer Institute for data collection. The Intramural Research Program of the National Institutes of Health, National Cancer Institute, Division of Cancer Epidemiology and Genetics, USA funded the current study.

\section{Conflict of interest}

The authors declare no conflict of interest.

Supplementary Information accompanies the paper on British Journal of Cancer website (http://www.nature.com/bjc) 


\section{REFERENCES}

Abe R, Yamagishi S (2008) AGE-RAGE system and carcinogenesis. Curr Pharm Des 14: $940-945$

Andreotti G, Chen J, Gao YT, Rashid A, Chang SC, Shen MC, Wang BS, Han TQ, Zhang BH, Danforth KN, Althuis MD, Hsing AW (2008) Serum lipid levels and the risk of biliary tract cancers and biliary stones: a population-based study in China. Int J Cancer 122: 2322-2329

Bastard JP, Maachi M, Lagathu C, Kim MJ, Caron M, Vidal H, Capeau J, Feve B (2006) Recent advances in the relationship between obesity, inflammation, and insulin resistance. Eur Cytokine Netw 17: 4-12

Cowey S, Hardy RW (2006) The metabolic syndrome: a high-risk state for cancer? Am J Pathol 169: $1505-1522$

de Luca C, Olefsky JM (2008) Inflammation and insulin resistance. FEBS Lett 582: $97-105$

Grundy SM, Cleeman JI, Daniels SR, Donato KA, Eckel RH, Franklin BA, Gordon DJ, Krauss RM, Savage PJ, Smith Jr SC, Spertus JA, Costa F (2005) Diagnosis and management of the metabolic syndrome: an American Heart Association/National Heart, Lung, and Blood Institute Scientific Statement. Circulation 112: $2735-2752$

Hsing AW, Gao YT, Han TQ, Rashid A, Sakoda LC, Wang BS, Shen MC, Zhang BH, Niwa S, Chen J, Fraumeni Jr JF (2007) Gallstones and the risk of biliary tract cancer: a population-based study in China. Br J Cancer 97: $1577-1582$

Hsing AW, Rashid A, Devesa SS, Fraumeni Jr JF (2006) Biliary tract cancer. In: Schottenfeld D, Fraumeni JF Jr (eds) Cancer Epidemiology and Prevention, 3rd edn. Oxford University Press, Inc.: New York, pp 787-800

Hsing AW, Sakoda LC, Rashid A, Chen J, Shen MC, Han TQ, Wang BS, Gao YT (2008) Body size and the risk of biliary tract cancer: a populationbased study in China. Br J Cancer 99: 811-815

Ishiguro S, Inoue M, Kurahashi N, Iwasaki M, Sasazuki S, Tsugane S (2008) Risk factors of biliary tract cancer in a large-scale population-based cohort study in Japan (JPHC study); with special focus on cholelithiasis, body mass index, and their effect modification. Cancer Causes Control 19: $33-41$

Jaiswal M, LaRusso NF, Shapiro RA, Billiar TR, Gores GJ (2001) Nitric oxide-mediated inhibition of DNA repair potentiates oxidative DNA damage in cholangiocytes. Gastroenterology 120: 190-199

Janowitz P, Wechsler JG, Kuhn K, Kratzer W, Tudyka J, Swobodnik W, Ditschuneit H (1992) The relationship between serum lipids, nucleation time, and biliary lipids in patients with gallstones. Clin Investig 70: $430-436$

Joint Committee for Developing Chinese Guidelines on Prevention and Treatment of Dyslipidemia in Adults (2007) Chinese guidelines on prevention and treatment of dyslipidemia in adults. Zhonghua Xin Xue Guan Bing Za Zhi 35: 390-419

Jonkers IJ, Smelt AH, Ledeboer M, Hollum ME, Biemond I, Kuipers F, Stellaard F, Boverhof R, Meinders AE, Lamers CH, Masclee AA (2003) Gall bladder dysmotility: a risk factor for gall stone formation in hypertriglyceridaemia and reversal on triglyceride lowering therapy by bezafibrate and fish oil. Gut 52: $109-115$

Kodama H, Kono S, Todoroki I, Honjo S, Sakurai Y, Wakabayashi K, Nishiwaki M, Hamada H, Nishikawa H, Koga H, Ogawa S, Nakagawa K
(1999) Gallstone disease risk in relation to body mass index and waist-to-hip ratio in Japanese men. Int J Obes Relat Metab Disord 23: $211-216$

Levy JC, Matthews DR, Hermans MP (1998) Correct homeostasis model assessment (HOMA) evaluation uses the computer program. Diabetes Care 21: $2191-2192$

Lyssenko V, Almgren P, Anevski D, Perfekt R, Lahti K, Nissen M, Isomaa B, Forsen B, Homstrom N, Saloranta C, Taskinen MR, Groop L, Tuomi T (2005) Predictors of and longitudinal changes in insulin sensitivity and secretion preceding onset of type 2 diabetes. Diabetes 54: 166-174

Maclure KM, Hayes KC, Colditz GA, Stampfer MJ, Speizer FE, Willett WC (1989) Weight, diet, and the risk of symptomatic gallstones in middleaged women. $N$ Engl J Med 321: $563-569$

Maurer KJ, Carey MC, Fox JG (2009) Roles of infection, inflammation, and the immune system in cholesterol gallstone formation. Gastroenterology 136: $425-440$

National Institutes of Health, National Heart, Lung, and Blood Institute (1995) National Cholesterol Education Program, Recommendations on Lipoprotein Measurement from the Working Group on Lipoprotein Measurement. NIH Publication No. 95-3044: Bethesda, MD

Olefsky JM, Glass CK (2010) Macrophages, inflammation, and insulin resistance. Annu Rev Physiol 72: 219-246

Rifai N, Warnick GR, McNamara JR, Belcher JD, Grinstead GF, Frantz Jr ID (1992) Measurement of low-density-lipoprotein cholesterol in serum: a status report. Clin Chem 38: $150-160$

Seidell JC (2010) Waist circumference and waist/hip ratio in relation to all-cause mortality, cancer and sleep apnea. Eur J Clin Nutr 64: $35-41$

Shebl FM, Andreotti G, Rashid A, Gao YT, Yu K, Shen MC, Wang BS, Li Q, Han TQ, Zhang BH, Fraumeni Jr JF, Hsing AW (2010) Diabetes in relation to biliary tract cancer and stones: a population-based study in Shanghai, China. Br J Cancer 103: $115-119$

Shor R, Wainstein J, Oz D, Boaz M, Matas Z, Fux A, Halabe A (2008) Low HDL levels and the risk of death, sepsis and malignancy. Clin Res Cardiol 97: $227-233$

Smith L, Lucas D, Lehnus G (1979) Automated measurement of total cholesterol and triglycerides, in 'tandem,' on the discrete sample analyzer, Gilford System 3500. Clin Chem 25: 439-442

Tabet F, Rye KA (2009) High-density lipoproteins, inflammation and oxidative stress. Clin Sci (Lond) 116: $87-98$

Tsai CJ, Leitzmann MF, Willett WC, Giovannucci EL (2004) Prospective study of abdominal adiposity and gallstone disease in US men. Am J Clin Nutr 80: $38-44$

WHO Expert Consultation (2004) Appropriate body-mass index for Asian populations and its implications for policy and intervention strategies. Lancet 363: $157-163$

WHO/IASO/IOTF (2000) The Asia-Pacific Perspective: Redefining Obesity and its Treatment. Health Communications Australia Pty Ltd: Australia, Melbourne, Full document available from http://www. wpro.who.int/NR/rdonlyres/0A35147B-B1D5-45A6-9FF2-F7D86608A4DE/ 0/Redefiningobesity.pdf

This work is published under the standard license to publish agreement. After 12 months the work will become freely available and the license terms will switch to a Creative Commons Attribution-NonCommercial-Share Alike 3.0 Unported License. 